http:/ / dx.doi.org/10.21707/gs.v11.n03a010

\title{
ANÁlise dA PAISAGEM APÓS SIMULAÇÃO DA CONSTRUÇÃO DA FERROVIA DE INTEGRAÇÃO OESTE LESTE EM CORREDOR ECOLÓGICO DA MATA Atrântica
}

\author{
Maria Eugênia Bruck de Moraes ${ }^{*}$, Lauro Brito de Santana ${ }^{2}$, Ronaldo Lima Gomes ${ }^{3}$
}

\author{
${ }^{1}$ Docente do Departamento de Ciências Agrárias e Ambientais (DCAA) e do Programa de Pós-Graduação em Desenvolvimento e Meio Ambiente \\ (PRODEMA), Universidade Estadual de Santa Cruz, (UESC). Rodovia Jorge Amado, Km 16, Ilhéus, Bahia, CEP $45662-900$. \\ ${ }^{2}$ Discente do Mestrado em Desenvolvimento e Meio Ambiente, Universidade Estadual de Santa Cruz. \\ ${ }^{3}$ Docente do Departamento de Ciências Agrárias e Ambientais (DCAA) e do Programa de Pós-Graduação em Desenvolvimento e Meio Ambiente \\ (PRODEMA), Universidade Estadual de Santa Cruz. \\ Autorpara correspondência: engeniabruck@uesc.br
}

Recebido em 29 de novembro de 2016. Aceito em 23 de junho de 2017. Publicado em 29 de julho de 2017.

Resumo - O artigo apresenta uma análise dos fragmentos florestais inseridos na área de influência do trecho final da Ferrovia Oeste-Leste que ligará Figueirópolis, no Tocantins à Ilhéus, na Bahia, a partir de métricas da paisagem e, após a simulação da sua construção, visando identificar as possíveis alterações nesses fragmentos. O mapeamento dos mesmos foi feito a partir de aerofotos, em escala 1:2.000, utilizando o Sistema de Informação Geográfica ArcGIS 10.2. Também foi calculado o valor de importância dos fragmentos àconservação ambiental. Inicialmente foram mapeados 79 fragmentos de cobertura arbórea dominante e de restinga arbórea. No entanto, com a simulação da construção da ferrovia, o número de fragmentos aumentou para 89, sendo que quase $60 \%$ possui entre 5 ha e 50 hae apenas 7,87\% mais de 250 ha. Quanto ao grau de vulnerabilidade ecológica relativa que representa a suscetibilidade ao efeito de borda, 62 fragmentos apresentaram alta vulnerabilidade e apenas sete, baixa. E quanto ao grau de isolamento dos fragmentos na paisagem, analisado a partir da simulação de sua conectividade, os resultados mostraram que a distância mínima para conectá-los seria de 300 metros. Conclui-se que dos 89 fragmentos, 7,87\% apresenta um valor de importância à conservação ambiental muito alto, enquanto 67,42\%um valor mediano. Mas esses últimos são essenciais na manutenção da conectividade funcional da paisagem, pois servem como fragmentos de ligação entre os fragmentos maiores. Assim, recomenda-se a adoção de estratégias de restauração florestal que possam minimizar os impactos da fragmentação que serão causados pela construção da ferrovia.

Palavras-chave: Ecologla da Pais agem; Fragmenta a Ão; Geoprocess amento; Corredor Central da Mata Atl Ântica.

LANDSCAPE ANALYSIS AFTER SIMULATION OF THE WEST-EAST INTEGRATION RAILROAD CONSTRUCTION IN THE ECOLOGICAL FOREST CORRIDOR OF THE ATLANTIC Forest

Aвstract - The article presents an analysis on the forest fragments inserted in the influence area at the final stretch of the West-East Railroad, which will connect Figueirópolis, Tocantis to Ilhéus, Bahia, through landscape metrics and, after its construction simulation, aiming to identify possible alterations in these fragments. The fragments mapping was done with the aid of air photos in a 1:2,000 scale, using the Geographical Information System ArcGIS 10.2. It was also calculated the importance value of the fragments face to environmental conservation. Initially, 79 fragments of dominant arboreal cover were mapped. However, after the railroad construction simulation, this number increased to 98 , in which almost 60\% having between 5 and 50ha and only $7.87 \%$ having more than 250 ha. In regards to the relative ecological vulnerability degree that represents the liability to the board effect, 62 fragments presented high vulnerability and only seven, low vulnerability. As for the degree of fragments' isolation in the landscape, analyzed since the simulations of its 
connectivity, the results showed that the minimum distance for connection would be 300 meters. The conclusion taken was that from the 89 fragments, $7.87 \%$ presented an importance value to environmental conservation very high, while $67.42 \%$ had a medium value. These last are essential in the maintenance of the landscape functional connectivity, for they serve as linking fragments between bigger ones. This way, it is recommended to adopt forest restauration strategies that can minimize the fragmentation impacts that will be caused by the railroadconstruction.

Keywords: LandSCAPE ECOLOGy; Fragmentation; GeOproCESSING; Central Corridor of the ATLANTIC Forest.

ANÁlisis del paisaje trasla simulación dela CONSTrucción de la Ferrovía de integración OEste-Este en CORREDOR ECOLÓGICO DEL BOSQUE ATLÁNTICO

RESUMEN - El artículo presenta un análisis de los fragmentos forestales localizados en el área de influencia del trecho final de la Ferrovía Oest-Este que unirá Figueirópolis, en Tocantins, a Ilhéus, enBahía, a partir de las métricas del paisaje, y después de la simulación de su construcción, buscando identificarlas posibles alteraciones en esos fragmentos. El mapeo de los mismos fue hecho a partir de aerofotos, en escala 1:2.000, utilizando el Sistema de Información Geográfica ArcGIS 10.2. También fue calculado el valor de importancia de los fragmentos para la conservación ambiental. Inicialmente fueron mapeados 79 fragmentos de cobertura arbórea dominante y de restinga arbórea. Sin embargo, con la simulación de la construcción de laferrovía, el número de fragmentos se elevó a 89 , siendo que casi el $60 \%$ posee entre 5 ha y 50 ha y solo el 7,87\% más de 250ha. Con relación al grado de vulnerabilidad ecológica relativa, que representa la susceptibilidad al efecto de borde, 62 fragmentos presentaron alta vulnerabilidady solo siete baja. En cuanto al grado de aislamiento de los fragmentos en el paisaje, analizado a partir de la simulación de su conectividad, los resultados mostraron que la distancia mínima para conectarlos sería de 300 metros. Se concluye que de los 89 fragmentos, el 7,87\%, presenta un valor muy alto en importancia para la conservación ambiental, mientras que el 67,42\% presentaun valor medio. Pero estos fragmentos son esenciales para mantener la conexión funcional del paisaje, sirviendo de fragmentos de unión entre los fragmentos mayores. Así, se recomienda la adopción de estrategias de restauración forestal que puedan minimizar los impactos dela fragmentación que serán causados por la construcción de la ferrovía.

Palabras clave: Ecología del Pais aje; Fr agmentación; Geoproces amiento; Corredor Central del Bosque Atlántico.

\section{INTRODUÇÃO}

A construção da Ferrovia de Integração Oeste Leste (FIOL) totaliza cerca de $1.500 \mathrm{~km}$ de extensão. Segundo o Relatório de Impacto Ambiental (RIMA) das obras de implantação da Ferrovia de Integração Oeste Leste (EF 334), entre Figueirópolis (TO) e Ilhéus (BA), o objetivo principal da ferrovia é alterar a matriz de transportes brasileira, substituindo, quando possível, o transporte rodoviário pelo transporte ferroviário nas trocas de cargas entre as regiões oeste, central e leste do Brasil (Bellia e Santos, 2009).

A construção da FIOL poderá causar diversos impactos ao meio ambiente. Segundo o RIMA, citado acima, o principal deles será o desmatamento da vegetação, necessário para o início das obras na área diretamente afetada, a principal área a ser impactada, que corresponde a uma faixa de largura média de $80 \mathrm{~m}$. Ainda segundo o RIMA, a ferrovia atravessará diversas áreas de vegetação nativa, sendo várias delas protetoras de córregos, rios e nascentes. Além disso, a retirada dessa vegetação poderá alterar o comportamento de animais silvestres ali existentes, prejudicando ou, às vezes, impossibilitando a sua permanência na área (Bellia e Santos, 2009).

A implantação da ferrovia e as modificações na vegetação próxima ao seu eixo constituirão uma barreira física ao movimento seguro dos animais silvestres, podendo provocar alterações no seu comportamento, como o isolamento e a dificuldade de reprodução que inicialmente poderá afetar a abundância dos indivíduos e, em 
última instância, a diminuição da diversidade de espécies em determinadas áreas. As modificações na paisagem causadas pela construção da ferroviapoderão ser expressivas e devem ser criteriosamente estudadas, para que seja possível compatibilizar os possíveis impactos negativos com medidas mitigadoras. Metzger (2001) chama a atenção para o fato de que o homem está na origem dos principais impactos ambientais, mas também é ele quem tem a competência para implantar tais medidas mitigadoras, no intuito de minimizar esses impactos.

A Ecologia da Paisagem como uma área de conhecimento de interface entre as ciências biológicas e geográficas - com seus princípios teóricos e métricas, tem desempenhado um importante papel na definição de estratégias para o uso e ocupação do solo de modo mais conservacionista. O surgimento de alterações numa paisagem natural, decorrente de intervenções antrópicas, geralmente altera a dinâmica populacional de muitas espécies da fauna e flora, visto que o aparecimento de uma nova matriz na paisagem (elemento dominante), como as áreas agrícolas e as pastagens, bem como de barreiras antrópicas representadas por as estradas e ferrovias, entre outras, interferem diretamente na dispersão de sementes e no deslocamento de algumas espécies animais que não sobrevivem em ambientes fragmentados.

No Brasil, o processo de fragmentação das paisagens aumentou significativamente nas últimas décadas, e a consequente perda de hábitats se configura como uma das maiores ameaças à conservação da biodiversidade (Tabarelli e Gascon, 2005). A fragmentação florestal, caracterizada por uma ruptura das unidades de paisagem (Metzger, 2003), frequentemente isoladas umas das outras (Primack e Rodrigues, 2006), encontra-se entre as mais graves ameaças para a manutenção dos ecossistemas e da diversidade biológica (Debinski e Holt, 2000), provocando ou acentuando o grau de isolamento de determinadas populações.

Os principais efeitos da fragmentação da paisagem, segundo Geneletti (2003) são: a diminuição do tamanho dos fragmentos e de suas áreas-núcleo, o aumento da suscetibilidade a distúrbios externos e aos efeitos de borda, a invasão de espécies exóticas e a alteração de suas características originais, como a mudança no formato dos fragmentos e na composição da biota. Nesse sentido, os efeitos nocivos do processo de fragmentação ultrapassam a supressão de hábitats, afetando a qualidade dos remanescentes florestais, onde espécies vegetais mais adaptadas às perturbações antrópicas, principalmente as pioneiras, se tornam espécies dominantes (Laurance et al., 1997), alterando a dinâmica e o equilíbrio dos ecossistemas. Geneletti (2004) ressalta que as ações voltadas à conservação ambiental devem seguir em direção oposta à da fragmentação, principalmente quando se vislumbra manter ou restaurar a biodiversidade em paisagens fragmentadas.

Herrmann (2011) coloca que uma das estratégias para se minimizar as consequências da fragmentação é investir em um sistema de conectividade da paisagem (landscape linkages) que pode ser tanto estrutural, quando está relacionada à configuração espacial da paisagem, quanto funcional, a qual depende da capacidade de dispersão e das respostas comportamentais dos organismos.

Acredita-se que a análise métrica dos fragmentos possa contribuir coma adoção de estratégias que priorizem o estabelecimento da conectividade da paisagem. Metzger (2001; 2003) salienta que a análise dos fragmentos a partir da adoção dos princípios da Ecologia da Paisagem e da aplicação de suas métricas tem como ponto central o reconhecimento da dependência espacial entre as unidades de paisagem, uma vez que o funcionamento de uma unidade depende das interações com as unidades vizinhas. Também se espera que a metodologia aqui aplicada possa servir de base para outros estudos semelhantes. Diante do exposto, no presente artigo apresenta-se uma análise dos fragmentos florestais inseridos na área de influência direta do traçado da FIOL, a partir da simulação de sua construção, visando identificar as possíveis alterações nos mesmos e na paisagem como um todo. 


\section{Material e MÉtodos}

Área de estudo

O trecho final do traçado da FIOL está projetado para passar por uma área considerada de alta biodiversidade e endemismo (Martini et al., 2007; Thomas et al., 2008),o Mini Corredor PESC - Boa Esperança, localizado na denominada Região Litoral Sul do Estado da Bahia, mais precisamente ao longo de uma faixa costeira de $18 \mathrm{~km}$ de largura que se estende desde a foz do Rio de Contas, em Itacaré, até as proximidades da cidade de Ilhéus, totalizando uma área de $1.375,14 \mathrm{~km}^{2}$. Esse corredor ecológico possui $63,34 \%$ de sua área inserida no município de Ilhéus, 25,84\% no município de Itacaré, 10,77\% no município de Uruçuca e, uma pequena parcela $(0,04 \%)$ no município de Itabuna (Gomes et al., 2012). O eixo da FIOL vai adentrar o Mini Corredor PESC - Boa Esperança e percorrer uma distância de $13,3 \mathrm{~km}$, margeando o rio Almada no município de Ilhéus (Figura 1).

Esse corredor ecológico faz parte do Corredor Central da Mata Atlântica e é formado por uma rede de áreas protegidas, como a Área de Proteção Ambiental (APA) Costa de Itacaré - Serra Grande criada pelo Decreto Estadual no 2.186 de 07 de junho de 1993 (BAHIA. Decreto 2.186/1993), a APA da Lagoa Encantada e do Rio Almada criada pelo Decreto Estadual no 2.217 de 14 de junho de 1993(BAHIA. Decreto 2.217/1993) e ampliada em 22 de setembro de 2003 pelo Decreto Estadual no 8.650 (BAHIA. Decreto 8.650/2003), o Parque Estadual da Serra do Conduru (PESC) criado pelo Decreto Estadual no 6.227 de 21 de fevereiro de 1997 (BAHIA. Decreto 6.227/1997), o Parque Municipal da Boa Esperança criado pela Lei Orgânica do Município de Ilhéus no dia 05 de abril de 1990 (ILHÉUS. Lei Orgânica/1990), o Parque Municipal Marinho dos Ilhéus criado pela Lei Municipal no 3.212 de 30 de janeiro de 2006 (ILHÉUS. Lei 3.212/2006) e o Parque Estadual Ponta da Tulha criado pelo Decreto Estadual no 16.487 de 22 de dezembro de 2015 (BAHIA. Decreto 16.487/2015) (Figura 1).

Em uma escala de paisagem, os remanescentes de mata atlântica encontram-se imersos numa matriz (elemento dominante) formada por um mosaico agrícola-urbano e, portanto, bastante fragmentado (Lira et al., 2012) e, em função das pressões antrópicas sobre tais remanescentes, apenas a criação de uma série de unidades de conservação não será suficiente para manter populações viáveis nessas áreas, especialmente nos fragmentos menores e isolados, que muitas vezes se encontram em diferentes estágios de sucessão (Fonseca et al., 2009; Ribeiro et al., 2009). Assim, por conta da relevância ambiental e da necessidade de se interligar tais unidades de conservação, se propôs a criação do Mini Corredor PESC - Boa Esperança, no âmbito do Projeto Corredor Central da Mata Atlântica, integrando a Área Focal Camamu-Cabruca-Conduru, um de seus trechos prioritários, em virtude de manter conexão, ao norte, com o Mini Corredor Serra das Onças e ao sul, com a Área Focal UnaLontras-Baixão.

Os corredores naturais são estruturas lineares que têm a função de conectar os elementos da paisagem (Forman e Collinge, 1997), facilitando os fluxos hídricos e biológicos. Os corredores ecológicos podem ser formados por um sistema de áreas protegidas interconectadas que tem por objetivo evitar o isolamento das mesmas. Atualmente, tais corredores têm sido adotados como uma política de gestão em rede das áreas protegidas (Herrmann, 2011).

No presente trabalho de pesquisa, adotou-se como área de estudo, a área de influência diretada construção da ferrovia, a qual corresponde a uma faixa de $10 \mathrm{~km}$ de largura, sendo $5 \mathrm{~km}$ para cada lado da via férrea, com 
um total de $158 \mathrm{~km}^{2}$. A escolha da área de estudo justifica-se em função de abranger áreas que potencialmente podem sofrer a influência da circulação de veículos durante ou após a construção da ferrovia e da poluição que as obras ou acidentes possam vir a causar, conforme consta no Estudo de Impacto Ambiental (EIA) das Obras de Implantação da Ferrovia Oeste Leste (EF334), entre Figueirópolis (TO) e Ilhéus (BA) (Bellia et al., 2009).

Figura 1 - Mapa de localização da área de estudo, área de influência direta da Ferrovia de Integração Oeste Leste (FIOL) no Mini Corredor PESC - Boa Esperança e no Estado da Bahia.

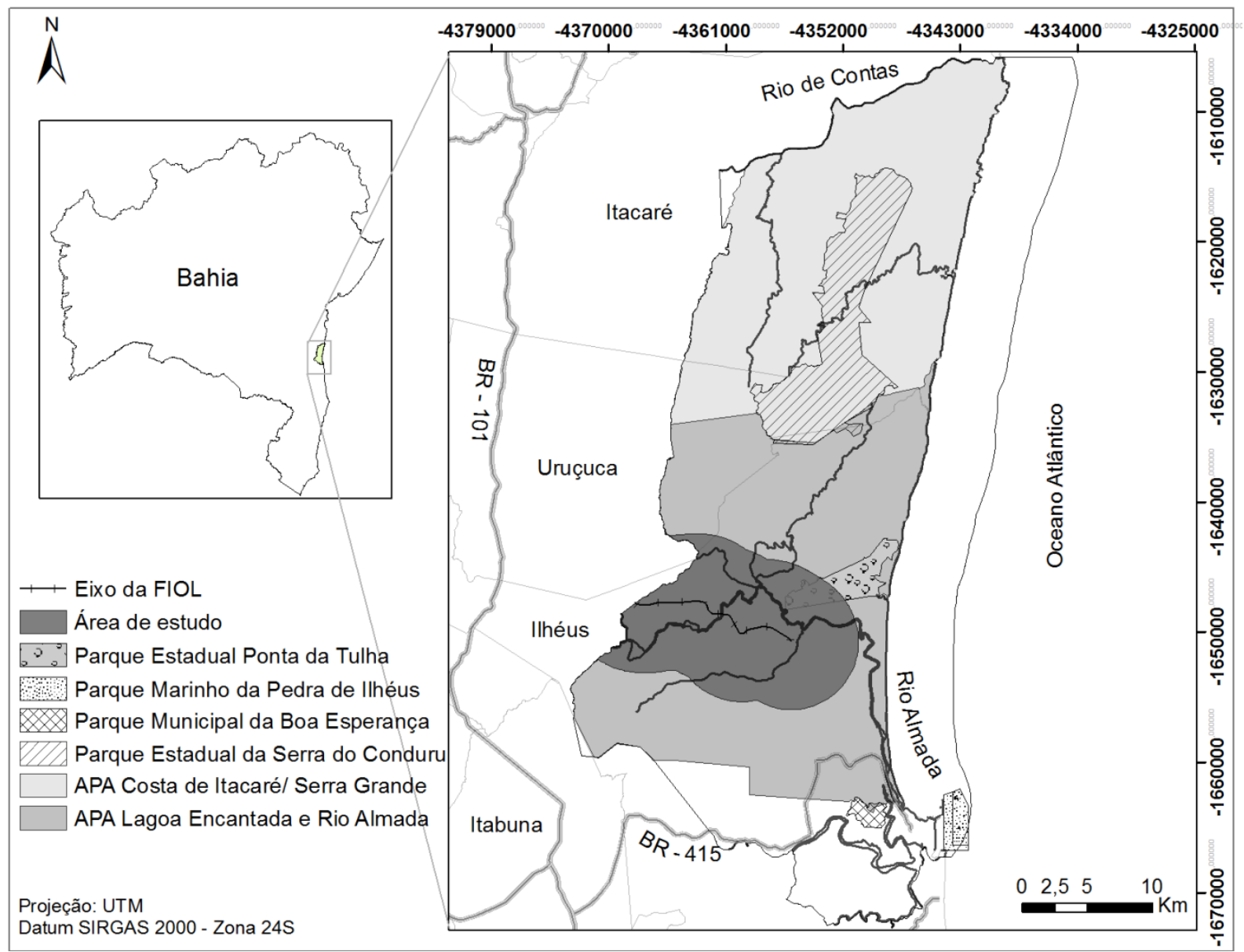

Fonte: Base de dados cartográficos da SEI (2016) / Gomes et al. (2012) / Bellia e Santos (2009) / Bellia et al. (2009).

Já, a área adotada para a simulação do traçado do eixo da FIOL corresponde àárea diretamente afetada, que corresponde a uma faixa de $80 \mathrm{~m}$ de largura, sendo40 m para cada lado, de acordo com o EIA citado anteriormente. Trata-se da área que sofrerá o impacto direto da execução das obras, em termos de poluição do ar, ruídos, vibrações, modificações na topografia, na drenagem superficial e supressão da vegetação original (Belliaet al., 2009).

\section{Metodologia}

O mapeamento dos fragmentos florestais existentes na área de estudo foi elaborado com o apoio do Laboratório de Análise e Planejamento Ambiental, a partir da interpretação de um mosaico de fotografias aéreas digitais de 2010, escala 1:2.000, disponibilizadas pelo Departamento de Infraestrutura de Transportes da Bahia 
(DERBA), utilizando-se o Sistema de Informação Geográfica (SIG) ArcGis 10.2, licenciado pelo Laboratório de Geoprocessamento da Base Ambiental Costa do Cacau.

A identificação das diferentes formas de cobertura vegetal arbórea-arbustivafoi feita com base na fisionomia das formações vegetais, que é um dos parâmetros para análise dos estágios sucessionais da mata atlântica no estado da Bahia, estabelecidospela Resolução CONAMA nº 05 de 04 de maio de 1994 (BRASIL. Resolução CONAMA 05/1994). Assim, foram mapeadosos fragmentos florestais das seguintes classes de cobertura vegetal:

- Cobertura arbórea dominante: com altura média superior aos $12 \mathrm{~m}$ e dossel fechado, corresponde ao estágio avançado de regeneração da floresta. Nas fotografias aéreas a cor predominante é o verde escuro e a textura é rugosa. Refere-se aos remanescentes de Floresta Ombrófila Densa e às áreas de "cacau-cabruca" abandonadas, um sistema agroflorestal típico da região cacaueira, onde o cacau (Theobroma cacao) é cultivado sob a copa das árvores da Floresta Ombrófila Densa (Sambuichiet al., 2012), sendo considerado um sistema agroflorestal que contribui para a conservação da biodiversidade no bioma da mata atlântica (Schroth et al., 2011). Na área de estudo, a partir dos trabalhos de campo realizados em janeiro de 2015, verificou-se que neste caso, trata-se de áreas onde o cultivo do cacau deixou de ser manejado e a floresta encontra-se em estágio avançado de regeneração.

- Restinga arbórea: com altura média de até $10 \mathrm{~m}$ e dossel fechado. No mosaico de aerofotos, a cor varia em tons de verde-médio e a textura é pouco rugosa. Essa classe de cobertura vegetal foi individualizada das demais a partir da sobreposição das fotografias aéreas com o mapa de solos elaborado por Gomes et al. (2012), o que permitiu a identificação das áreas de vegetação arbórea sobre solos arenosos, as quais foram checadas com base em um trabalho de campotambém realizado em janeiro de 2015.

Os fragmentos florestais foram mapeados priorizando-se aqueles com cobertura arbórea-arbustiva acima de 3 ha, com base no exposto por Laurance et al. (1997). Já, a simulação do traçado da ferrovia, apresentado no EIA, foi feita a partir da digitalização em tela do seu eixo, também usando o ArcGis 10.2. Em seguida foi suprimida a cobertura vegetal existente naárea diretamente afetada, por se considerar que tal vegetação, provavelmente será desmatada para a construção da ferrovia. Posteriormente, foi realizada aclassificação dos fragmentos de cobertura arbórea dominante e restinga arbórea em classes de tamanho em hectares.

A análise do grau de Vulnerabilidade Ecológica Relativa (VER), entendido como o grau de exposição do fragmento aos efeitos de borda foi realizada a partir da classificação dos fragmentos conforme a razão entre área-núcleo/área de borda, a qual foi delimitada considerando-se uma faixa de $30 \mathrm{~m}$ para o interior de cada fragmento, sendo o restante considerado como área-núcleo, de acordo com o proposto por Pires et al. (2004). Ainda com base no proposto por tais autores, foram considerados com baixo grau à VER, os fragmentos maiores que 100 ha e com relação área-núcleo/área de borda maior que 5; os fragmentos com relação áreanúcleo/área de borda menor que 2,5 foram considerados como de alto grau à VER, eaqueles com relação áreanúcleo/área de borda entre 2,5 e 5, foram considerados fragmentos com médio grau à VER.

A análise do grau de isolamento dos fragmentos foi realizada através da expansão de suas bordas em distâncias previamente determinadas, que variaram de $50 \mathrm{~m}$ a 300 m, segundo proposta de Rantaet al. (1998). Os fragmentos foram considerados mais isolados quando a uma distância (d) pré-determinada não ocorreu sobreposição de sua borda à do(s) fragmento(s) mais próximo(s), da mesma maneira que, o fragmento foi considerado pouco isolado quando houve sobreposição de sua borda expandida em relação à borda expandida do(s) fragmento(s) vizinho(s). 
A análise do Valor de Importância à Conservação Ambiental (VICA) dos fragmentos foi realizada a partir de uma adaptação da metodologia proposta por Duriganet al. (2009), que permitiu a identificação do VICA para cada fragmento de cobertura arbórea dominante e restinga arbórea mapeados, a partir da adoção de 4 indicadores: tamanho, proteção de cursos d'água e/ou nascentes, grau de isolamento e grau de VER.

Os indicadores foram ponderados e compostos por uma série de fatores, sendo que cada fragmento foi analisado individualmente, recebendo uma nota de acordo com o fator considerado (Tabela 1). Cada nota foi multiplicada pelo peso do indicador e seu somatório representou o VICA.

Tabela 1 - Indicadores, pesos, fatores e notas utilizados na análise do Valor de Importância à Conservação Ambiental (VICA) dos fragmentos de cobertura arbórea dominante e de restinga arbórea.

\begin{tabular}{|c|c|c|c|}
\hline Indicadores & Pesos & Fatores & Notas \\
\hline \multirow{4}{*}{ Tamanho } & \multirow{4}{*}{4} & Fragmentos $<3 \mathrm{ha}$ & 1 \\
\hline & & Fragmentos entre 3 e $25 \mathrm{ha}$ & 2 \\
\hline & & Fragmentos entre 25 e 250 ha & 3 \\
\hline & & Fragmentos $>250 \mathrm{ha}$ & 4 \\
\hline \multirow{3}{*}{ Proteção de corpos d'água } & \multirow{3}{*}{4} & Sem curso d'água e nascentes & 1 \\
\hline & & Com curso d'água e sem nascentes & 2 \\
\hline & & Com cursos d'água e nascentes & 3 \\
\hline \multirow{3}{*}{ Grau de isolamento } & \multirow{3}{*}{3} & Alto (distância média 200 m) & 1 \\
\hline & & Médio (distância média ${ }^{3} 150<200 \mathrm{~m}$ ) & 2 \\
\hline & & Baixo (distância média ${ }^{3} 50<150$ m) & 3 \\
\hline \multirow{3}{*}{ Grau de VER* } & \multirow{3}{*}{2} & Alto $<2,5$ & 1 \\
\hline & & Médio ${ }^{3} 2,5 £, 5$ & 2 \\
\hline & & Baixo $>5$ & 3 \\
\hline
\end{tabular}

* VER - Vulnerabilidade Ecológica Relativa

Fonte: Adaptado de Duriganet al. (2009).

\section{RESUlTADOS E DISCUSSÃO}

Para Viana et al. (1992), a distribuição dos fragmentos em classes de tamanho é de suma importância para o desenvolvimento de estratégias de conservação ambiental e a tomada de decisão.Desse modo, na tabela 2 apresenta-se a classificação dos fragmentos de cobertura arbórea dominante e de restinga arbórea na área de estudo, sem e com a simulação da construção da FIOL.

Inicialmente, foram mapeados 79 fragmentos de cobertura arbórea dominante e de restinga arbórea. Porém, com a simulação da construção do trecho final da FIOL na área de estudo, a quantidade de fragmentos passou para 89 (Tabela 2) com o aumento de 10 fragmentos, muitos deles com menos de 3 ha.

Salienta-se que autores como Laurance et al. (1997) ressaltam que fragmentos muito pequenos (abaixo de 3 ha) contribuem muito pouco para a conservação da biodiversidade, uma vez que praticamente não possuem áreanúcleo, estando totalmente suscetíveis aos efeitos de borda. Esta alteração no número de fragmentos gerou um aumento de 12,66\% no número de fragmentos. Caso se confirme a supressão da vegetação na área diretamente afetada, também haverá e uma redução de 92,85 ha, principalmente de cobertura arbórea dominante. 
Tabela 2 - Classes de tamanho dos fragmentos de cobertura arbórea dominante e restinga arbórea na área de influência direta da Ferrovia de Integração Oeste Leste (FIOL), após a simulação de sua construção.

\begin{tabular}{lcccc}
\hline $\begin{array}{l}\text { Classes de } \\
\text { tamanho (ha) }\end{array}$ & $\begin{array}{c}\left.\text { Fragmentos } \mathbf{( n}^{\mathbf{0}}\right) \\
\text { sem a FIOL }\end{array}$ & $\begin{array}{c}\text { Área (ha) dos } \\
\text { fragmentos }\end{array}$ & $\begin{array}{c}\text { Fragmentos } \mathbf{( n}^{\mathbf{0}} \text { ) } \\
\text { com a FIOL }\end{array}$ & $\begin{array}{c}\text { Área (ha) dos } \\
\text { fragmentos }\end{array}$ \\
\hline$>3$ & --- & --- & 09 & 04,05 \\
$\geq 3<5$ & 09 & 36,78 & 07 & 28,03 \\
$\geq 5<10$ & 15 & 118,36 & 17 & 135,40 \\
$\geq 10<25$ & 21 & 302,10 & 23 & 351,30 \\
$\geq 25<50$ & 12 & 397,10 & 12 & 383,00 \\
$\geq 50<100$ & 08 & 591,01 & 07 & 505,50 \\
$\geq 100<250$ & 07 & $1.194,46$ & 07 & $1.194,40$ \\
$\geq 250$ & 07 & $4.602,52$ & 07 & $4.547,80$ \\
Total & $\mathbf{7 9}$ & $\mathbf{7 . 2 4 2 , 3 3}$ & $\mathbf{8 9}$ & $\mathbf{1 7 . 1 4 9 , 4 8}$ \\
\hline
\end{tabular}

Fonte: Dados da pesquisa classificados a partir da adaptação do trabalho de Pires et al. (2004).

Observando-se a Tabela 2, pode-se constatar que embora os fragmentos maiores (com mais de $250 \mathrm{ha}$ ) representem um número menor de fragmento (sete), estes possuem uma área de mais de 4.500 ha, mesmo havendo uma redução em torno de 54 ha com a simulação da construção da ferrovia, o que representa mais de 63\% da cobertura florestal existente na área de estudo e ressalta a importância dos mesmos.

Após a simulação da FIOL na área de estudo foi verificado que 62 fragmentos (perfazendo um total de 644,37 ha) passariam a apresentar alto grau de VER (Tabela 3), enquanto que sem a simulação (situação atual) 58 fragmentos, com um total 1.064,72 ha (o que representa uma redução de área de 420,35 ha) apresentam esse valor de vulnerabilidade.

Tabela 3 - Vulnerabilidade Ecológica Relativa (VER) dos fragmentos de cobertura arbórea dominante e restinga arbórea na área de influência direta da Ferrovia de Integração Oeste Leste (FIOL), antes e após a simulação de sua construção.

\begin{tabular}{lcccc}
\hline Grau de VER & $\begin{array}{c}\text { Fragmentos }\left(\mathbf{n}^{\mathbf{0}}\right) \\
\text { sem a FIOL }\end{array}$ & $\begin{array}{c}\text { Área dos } \\
\text { fragmentos (ha) }\end{array}$ & $\begin{array}{c}\text { Fragmentos }\left(\mathbf{n}^{\mathbf{0}}\right) \\
\text { com a FIOL }\end{array}$ & $\begin{array}{c}\text { Área dos } \\
\text { fragmentos (ha) }\end{array}$ \\
\hline Alto $<2,5$ & 58 & $1.064,72$ & 62 & 644,37 \\
Médio $\geq 2,5 \leq 5$ & 14 & $1.575,10$ & 13 & $1.156,19$ \\
Baixo $>5$ & 07 & $4.602,52$ & 07 & $4.030,77$ \\
Total & $\mathbf{7 9}$ & $\mathbf{7 . 2 4 2 , 3 4}$ & $\mathbf{8 2}$ & $\mathbf{5 . 8 3 1 , 3 3}$ \\
\hline
\end{tabular}

Fonte: Dados da pesquisa classificados a partir da adaptação do trabalho de Pires et al. (2004).

Os dados da Tabela 3 também demonstram que apenas sete fragmentos continuariam a apresentar baixo grau de VER, apesar de haver um decréscimo na área total de 571,75 ha. Além disso, com a possível divisão dos fragmentos mais próximos ao eixo da ferrovia, sete fragmentos passariam a apresentar área-núcleo inferior ao tamanho necessário para o cálculo do grau de VER, portanto apenas 82 fragmentos foram avaliados por essa métrica. Em contrapartida, esses sete fragmentos sozinhos continuariam a contabilizar mais de 4.000 ha, oque justifica a necessidade de preservação dos mesmos.

$\mathrm{Na}$ Figura 2, observa-se a distribuição espacial dos fragmentos conforme o seu grau de vulnerabilidade, 
bem como os fragmentos que serão divididos em dois - na melhor das hipóteses, com a construção do trecho final da FIOL em um corredor ecológico de importância ímpar para a conservação de espécies da mata atlântica.

Figura 2 - Distribuição espacial dos fragmentos de cobertura arbórea dominante e restinga arbórea por grau de vulnerabilidade ecológica relativa após a simulação da construção da Ferrovia de Integração Oeste Leste (FIOL) na área de estudo.

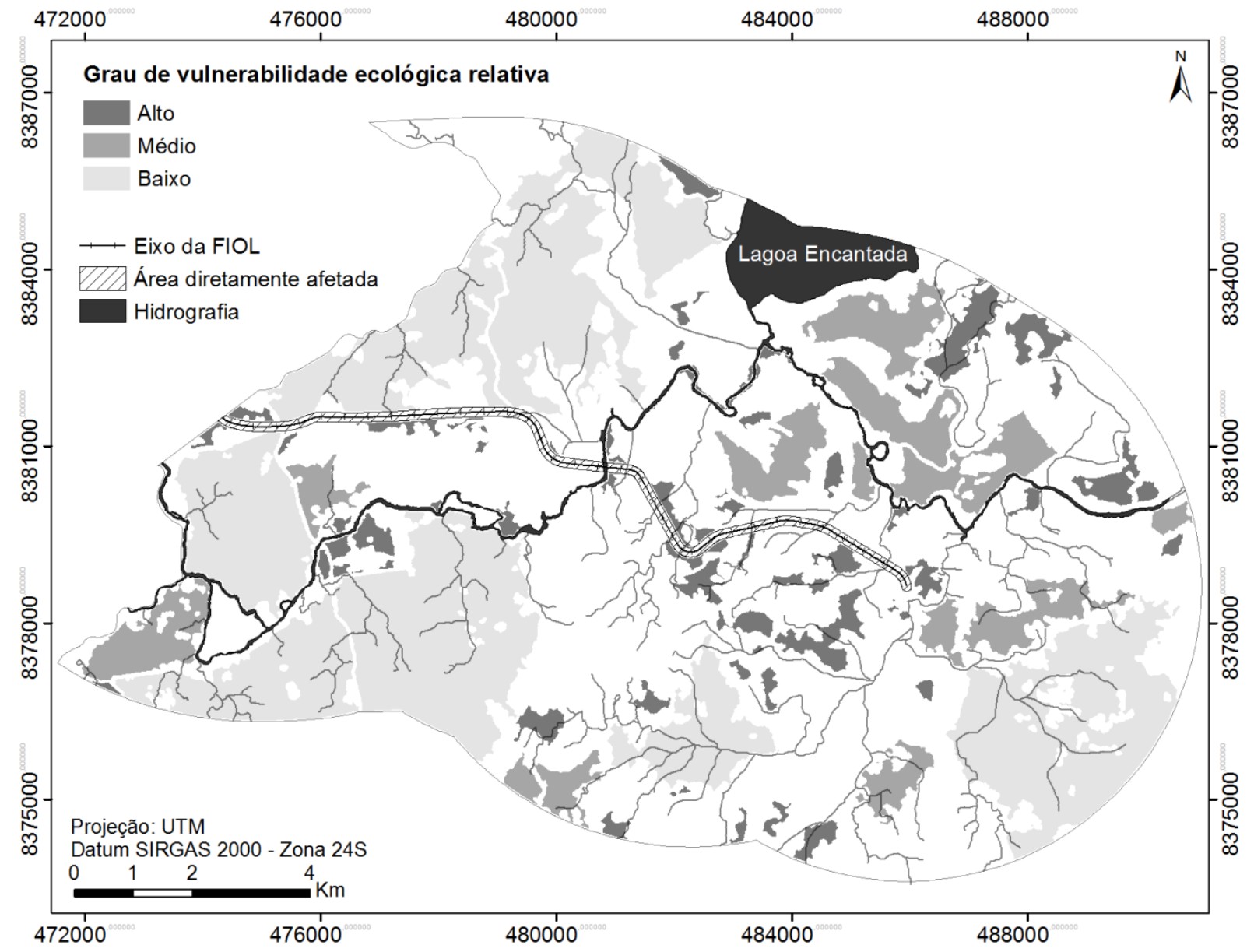

Fonte: Elaborado pelos autores a partir dos dados da pesquisa e fotografias aéreas digitais fornecidas pelo Departamento de Infraestrutura de Transportes da Bahia (2010).

$\mathrm{Na}$ avaliação do grau de isolamento dos fragmentos, considera-se que quanto menor a distância entre dois fragmentos, maior a possibilidade de movimentação da biota e o aumento do fluxo gênico. Enquanto que distâncias maiores implicam tanto no isolamento estrutural quanto funcional da paisagem. Silva e Souza (2014) destacam que a fragmentação florestal e os sucessivos efeitos de borda tendem a aumentar o isolamento das espécies e, podem ainda, resultar no desaparecimento de fragmentos menores. O grau de isolamentodos fragmentos na paisagem, analisado neste estudo, a partir da expansão de borda dos fragmentos, antes e após a simulação da construção da ferrovia, variou entre 50 me $300 \mathrm{~m}$ (Tabela 4).

Com a simulação da FIOL, houve um aumento no número de fragmentos conectados (58) com uma distância mínima de $50 \mathrm{~m}$, quando comparado com a mesma expansão de borda para o número de fragmentos atual (54), isto é, sem a construção da ferrovia. Por outro lado, a distância mínima necessária para conectar todos os fragmentospassou de 250 mpara $300 \mathrm{~m}$ (Tabela 4), o que demonstra um aumento no grau de isolamento dos fragmentos, caso a ferrovia seja implantada na área de estudo. 
Tabela 4 - Simulação da conexão dos fragmentos de cobertura arbórea dominante e restinga arbórea na área de influência direta da Ferrovia de Integração Oeste Leste (FIOL), antes e após a simulação de sua construção.

\begin{tabular}{ccc}
\hline $\begin{array}{c}\text { Distância da borda } \\
\text { expandida }(d)\end{array}$ & $\begin{array}{c}\mathbf{N}^{\mathbf{o}} \text { de fragmentos } \\
\text { conectados sem a } \\
\text { FIOL }\end{array}$ & $\begin{array}{c}\mathbf{N}^{\mathbf{o}} \text { de fragmentos } \\
\text { conectados com a } \\
\text { FIOL }\end{array}$ \\
\hline $50 \mathrm{~m}$ & 54 & 58 \\
$100 \mathrm{~m}$ & 67 & 71 \\
$150 \mathrm{~m}$ & 72 & 79 \\
$200 \mathrm{~m}$ & 77 & 85 \\
$250 \mathrm{~m}$ & 79 & 87 \\
$300 \mathrm{~m}$ & 79 & 89 \\
\hline
\end{tabular}

Fonte: Dados da pesquisa classificados a partir da adaptação do trabalho de Rantaet al. (1998).

Ao se comparar o grau de isolamento dos fragmentos com outros estudos que aplicaram a mesma metodologia em área de mata atlântica (Moraes et al., 2012; Moraes et al., 2015; Viana e Moraes, 2016), pode-se constatar que na área do presente estudo, a possibilidade de conectividade da paisagem é mais viável, uma vez que os fragmentos estão menos isolados e, ainda se pode considerar a contribuição do sistema agroflorestal “cacaucabruca", que mesmo quando manejado, desempenha um papel ecológico importante quando se configura como a matriz da paisagem, ao permitir a sua permeabilidade e contribuir com a conectividade funcional (Sambuichi et al., 2012).

No litoral sul da Bahia, a fragmentação da paisagem, decorrente das atividades antrópicas atreladas ao crescimento econômico,tem implicadono desmatamento da cobertura vegetal e, consequentemente, na diminuição de áreas propícias à conservação ambiental.Com o intuito de identificar os fragmentos mais significativos para a conservação, caso a construção do trecho final da FIOL seja finalizada, foi calculado o VICA de cada fragmento (Tabela 5).

Tabela 5 - Resultados do Valor de Importância à Conservação Ambiental (VICA) dos fragmentos de cobertura arbórea dominante erestinga arbórea, após a simulação da Ferrovia de Integração Oeste Leste na área de estudo.

\begin{tabular}{lcccc}
\hline VICA & Fragmentos $\left.\mathbf{( n}^{\mathbf{0}}\right)$ & Fragmentos (\%) & Área (ha) & Área (\%) \\
\cline { 3 - 5 } Médio $(14-27)$ & 60 & 67,42 & 662,10 & 09,26 \\
Alto $(28-38)$ & 22 & 24,72 & $1.939,58$ & 27,13 \\
Muito alto $(39-43)$ & 07 & 07,87 & $4.547,80$ & 63,61 \\
\hline Total & $\mathbf{8 9}$ & $\mathbf{1 0 0 , 0 0}$ & $\mathbf{7 . 1 4 9 , 4 8}$ & $\mathbf{1 0 0 , 0 0}$ \\
\hline
\end{tabular}

Fonte: Dados da pesquisa classificados a partir da adaptação do trabalho de Duriganet al. (2009).

De acordo com os dados apresentados na tabela 5, verifica-se que neste contexto, a área de estudo teria $67,42 \%$ dos seus fragmentos com médio VICA, 24,72\% com alto VICA e 7,87\% apresentaria VICA muito alto, sendo que nenhum fragmento apresentou baixo VICA.

Segundo Faith e Walker (1996), a decisão sobre a delimitação de áreas prioritárias à conservação ambiental deve considerar também a relação entre o custo e o risco para o investimento em conservação. Durigan et al. (2009) também relatam que, pode ser mais eficaz para a conservação uma estratégia que canalize recursos 
baseada em baixo custo e/ou baixo risco; salvo mediante uma justificativa excepcional,como por exemplo,para compensação por danos ambientais, como é o caso da área de estudo, que demanda atenção especial e a adoção de estratégias de restauração florestal que apresentem resultados positivos em um curto período de tempo.

A partir da análise da figura 3 é possível visualizar os fragmentos com muito alto VICA, sendo que dois desses fragmentos, aqueles mais próximos do trecho inicial do eixo da FIOL na área de estudo, possivelmente serão cortados pela ferrovia, o que diminuirá sua área-núcleo e, consequentemente, aumentará a sua área de borda, tornando-os mais suscetíveis a desaparecerem. Do mesmo modo, outro fragmento com muito alto VICA muito alto, provavelmente será totalmente desmatado para a construção do Porto Sul, que tem por objetivo escoar o minério de férreo que será transportado pela ferrovia (Belliaet al., 2009).

Figura 3 - Distribuição espacial dos fragmentos de cobertura arbórea dominante e restinga arbórea por grau do valor de importância à conservação ambiental após a simulação da construção da Ferrovia de Integração Oeste Leste (FIOL).

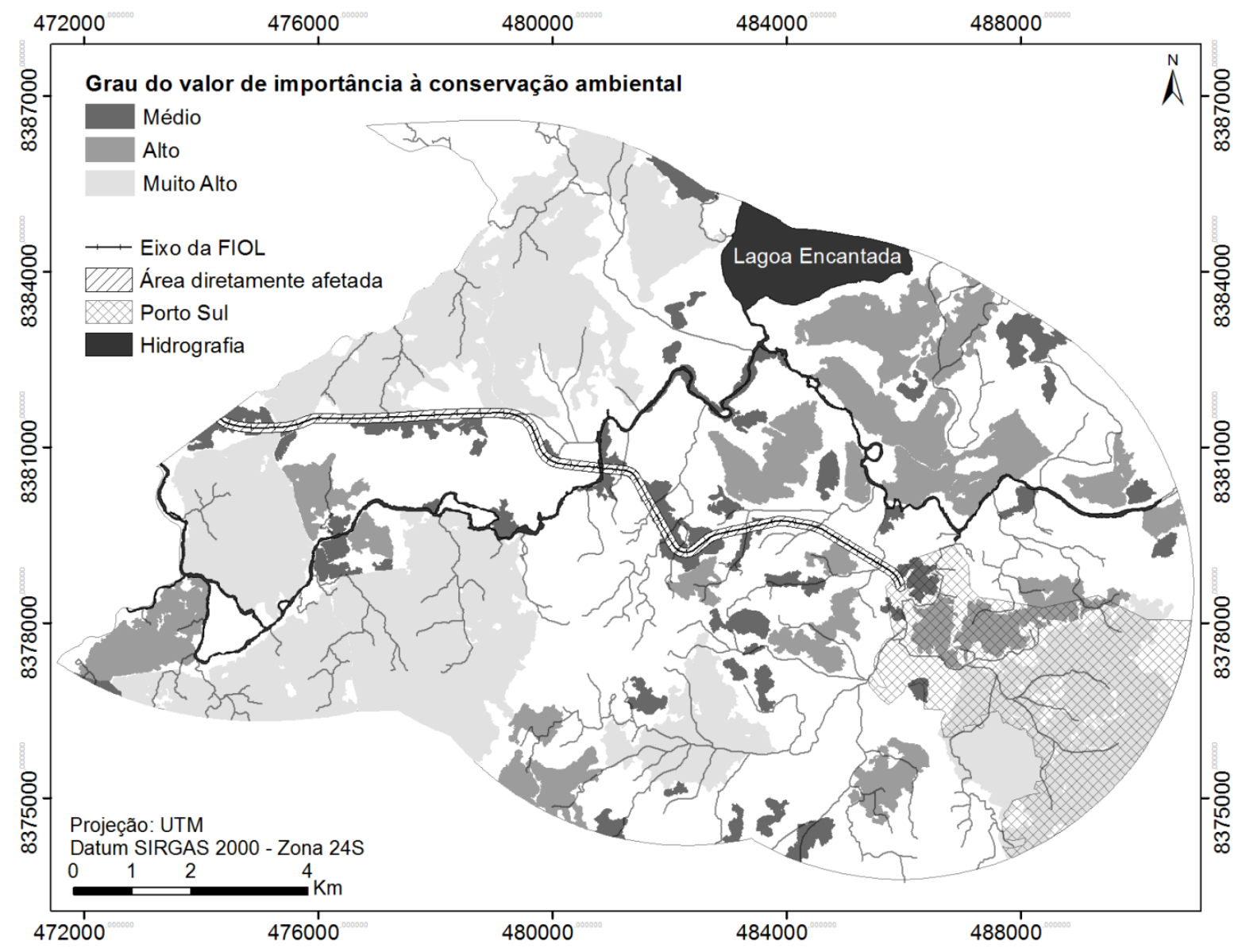

Fonte: Elaborado pelos autores a partir dos dados da pesquisa e fotografias aéreas digitais fornecidas pelo Departamento de Infraestrutura de Transportes da Bahia (2010).

Deste modo, na área em estudo, destacam-se quatro grandes fragmentos, com VICA muito alto e, considerados com maior potencial para a conservação, pois conforme destaca Cerqueira (2003), grandes manchas de hábitat com populações permanentes servem de área-fonte para recolonizar manchas menores. Porém, vale ressaltar que isso não quer dizer que os fragmentos menores e com médio e alto VICA não são importantes; uma vez que a depender da sua disposição espacial na paisagem, eles podem exercer a função de fragmentos de ligação (steppingstones) para espécies que migram e/ou se locomovem para fragmentos próximos; logo, devem ser incluídos em estratégias que visam à conectividade da paisagem que ao contrário da fragmentação, permitem o 
fluxo genético e o efeito resgate (Herrmann, 2011).

Apesar de se ressaltar a importância dos fragmentos aqui avaliados para a conservação dos recursos hídricos e da biodiversidade, torna-se necessário avaliar outros fatores como a qualidade dos mesmos a partir de inventários de fauna e flora. Ainda se destaca que a preservação possui interdependência com estrutura, riqueza de hábitats, grau de isolamento, natureza do entorno, relações interespecíficas e intraespecíficas, incluindo o comportamento social de animais e interferências antrópicas (Rambaldi e Oliveira, 2003), o que justifica a realização de estudos mais específicos, com a finalidade de melhor avaliar o estado de conservação dos fragmentos florestais antes da construção da ferrovia na áreade estudo.

\section{Conclusão}

A implantação da FIOL na área de estudo modificará o atual mosaico da paisagem na área de estudo, sendo que o maior impacto incidirá sobre os fragmentos de cobertura arbórea dominante e restinga arbórea, visto que vários deles serão atravessados pela ferrovia, o que irá implicar no aumento de 10 novos fragmentos, com menor área e alta vulnerabilidade relativa.

O grau de isolamento na paisagem, considerando-se a distância entre vizinhos mais próximos, variou de 50 a $300 \mathrm{~m}$, apontando alto índice de isolamento, podendo levar ao desaparecimento dos fragmentos menores, além de dificultar a troca de material genético entre as populações que acabarão isoladas.

As métricas de paisagem adotadas no estudo foram consideradas adequadas e essenciais na análise da área de estudo, apontando os fragmentos com maior valor de importância à conservação ambiental e aqueles que correm o risco de desaparecerem, o que pode servir de subsídios para a criação e implantação de estratégias de conservação e restauração da conectividade estrutural e funcional dos remanescentes de mata atlântica na área de estudo.

Para aprimorar a elaboração de tais estratégias, sugere-se que recursos e esforços sejam investidos em inventários biológicos dos fragmentos e, também, na sistematização desses dados em bases cartográficas detalhadas e georreferenciadas.

\section{Agradecimentos}

Os autores agradecem o apoio da Fundação de Amparo à Pesquisa do Estado da Bahia (FAPESB) e da Secretaria do Meio Ambiente do Estado da Bahia (SEMA) pelo financiamento do projeto (Convênio FAPESB / SEMA no 006/2013; Termo de Outorga PAM009/2014); do Programa de Pós-Graduação em Desenvolvimento e Meio Ambiente, do Laboratório de Análise e Planejamento Ambiental e do Laboratório de Geoprocessamento da Base Ambiental Costa do Cacau, da Universidade Estadual de Santa Cruz e à FAPESB pela bolsa de mestrado concedida ao segundo autor.

\section{REFERÊNCIAS}

BAHIA. Decreto 2.186, de 07 de junho de 1993. Dispõe sobre a criação da Área de Proteção Ambiental Costa 
de Itacaré-Serra Grande. Disponível em: http:/ / governo-ba.jusbrasil.com.br/legislacao/83183/decreto-2186-93 Acesso em: 29 nov. 2016.

BAHIA. Decreto 2.217, de 14 de junho de 1993. Dispõe sobre a criação da Área de Proteção Ambiental da Lagoa Encantada. Disponível em: http://governo-ba.jusbrasil.com.br/legislacao/83214/decreto-2217-93 Acesso em: 29 nov. 2016.

BAHIA. Decreto 6.227, de 21 de fevereiro de 1997. Dispõe sobre a criação do Parque Estadual da Serra do Conduru. Disponível em: http://governo-ba.jusbrasil.com.br/legislacao/79038/decreto-6227-97_Acesso em: 29 nov. 2016.

BAHIA. Decreto 8.650, de 22 de setembro de 2003. Dispõe sobre a ampliação da Área de Proteção Ambiental da Lagoa Encantada e do Rio Almada e dá outras providências. Disponível em: https://governo-ba.jusbrasil. com.br/legislacao/77566/decreto-8650-03 Acesso em: 29 nov. 2016.

BAHIA. Decreto 16.487, de 22 de dezembro de 2015. Dispõe sobre a criação do Parque Estadual Ponta da Tulha. Salvador: Diário Oficial do Estado da Bahia.

Bellia V e Santos LF. 2009. Relatório de Impacto Ambiental (RIMA) da Ferrovia de Integração Oeste Leste (EF 334) entre Figueirópolis (TO) e Ilhéus (BA). Rio de Janeiro: OIKOS Pesquisa Aplicada LTDA. Volume único.

Bellia V et al. 2009. Estudo de Impacto Ambiental (EIA) das obras de implantação da Ferrovia Oeste Leste (EF 334) entre Figueirópolis (TO) e Ilhéus (BA). Rio de Janeiro: OIKOS Pesquisa Aplicada LTDA. Volumes 1, 2 e 3.

Bennett AF e Saunders DA. 2010. Habitat fragmentation and landscape change. In: Sodhi NS e Ehrlich PR (Eds), Conservation Biology for All. New York: Oxford University Press, p. 88-106.

BRASIL. Resolução CONAMA 05, de 04 de maio de 1994. Definevegetação primária e secundária nos estágios iniciais, médio e avançado de regeneração da Mata Atlântica, a fim de orientar os procedimentos de licenciamento de atividades florestais no Estado da Bahia. Disponível em: http://www.mma.gov.br/port./ conama/legiabre.codlegi=146_Acesso em: 29 nov. 2016.

Cerqueira R et al. 2003. Fragmentação: alguns conceitos.In: Ministério do Meio Ambiente (Ed), Fragmentação de ecossistemas:causas, efeitos sobre a biodiversidade e recomendações de políticas públicas. Brasília: Ministério do Meio Ambiente, p. 21-40.

Debinski ID e Holt R. 2000. A survey and overview of habitats fragmentation. Conservation Biology,14(2):342-355.

Durigan Get al.2009. Protocolo de avaliação de áreas prioritárias para a conservação da mata atlântica na região da Serra do Mar/Paranapiacaba. Revista do Instituto Florestal, São Paulo, 21(1): 39-54.

Faith DP e Walker PA. 1996. Integrating conservation and development: effective trade-offs between biodiversity and cost in the selection of protected areas. Biodiversity and Conservation, 5(4):431-446. 
Fonseca CR et al. 2009. Towards an ecologically sustainable forestry in the Atlantic Forest.Biological Conservation,142(6):1209-1219.

Forman RTT e Collinge SK. 1997.Nature conserved in changing landscapes with and without spatial planning. Landscape and Urban Planning, 37(1-2):129-135.

Geneletti D. 2003. Biodiversity impact assessment of roads: an approach based on ecosystem rarity. Environmental Impact Assessment Review, 23(3):343-365.

Geneletti D. 2004. Using spatial indicators and value functions to assess ecosystem fragmentation caused by linear infrastructure. International Journal of Applied Earth Observation and Geoinformation, 5(1):1-15.

Gomes RL et al. 2012.Geoprocessamento aplicado ao planejamento da paisagem do minicorredor ecológico PESC - Boa Esperança. Ilhéus (BA): Instituto Floresta Viva / Universidade Estadual de Santa Cruz.Relatório final. Volume único.

Herrmann G. 2011. A origem do modelo corredor ecológico como ferramenta para o planejamento regional da conservação. In: Herrmann G. (Ed),Incorporando a teoria ao planejamento regional da conservação: a experiência do corredor ecológico da Mantiqueira. Belo Horizonte: Valor Natura. p. 5-46.

ILHÉUS. Lei Orgânica do Município de Ilhéus, de 05 de abril de 1990. Dispõe sobre a criação do Parque Municipal da Boa Esperança e dá outras providências. Disponível em: https://leismunicipais.com.br/leiorganica-ilheus-ba-1990-04-05-versao-compilada.html Acesso em: 29 nov. 2016.

ILHÉUS. Lei 3.212, de 30 de janeiro de 2006. Cria o Parque Marinho da Pedra de Ilhéus. Disponível em: http:// www.camara.ilheus.ba.io.org.br/diarioOficial/download/3212 Acesso em: 29 nov. 2016.

Laurance WF et al. 1997.Tropical forest fragmentation: synthesis of a diverse and dynamic discipline. In: Laurance WF e Bierregaard Jr RO (Eds), Tropical forest remnants: ecology, management, and coservation of fragmented communities. Chicago: University of Chicago Press, p. 502-525.

Lira PK et al. 2012. Land-use and land-cover change in Atlantic Forest landscapes. Forest Ecology and Management, 278: 80-89.

Martini AMZ et al. 2007. A hot-point within a hot-spot: a high diversity site in Brazil's Atlantic Forest. Biodiversity and Conservation,16(11): 3111-3128.

Metzger JP. 2001. O que é ecologia de paisagens? Biota Neotropica, Campinas (SP), 1(1-2):1-9.

Metzger JP. 2003. Como restaurar a conectividade de paisagens fragmentadas? In: KageyamaPYet al. (Eds), Restauração ecológica de ecossistemas naturais. Botucatu: FEPAF, p.51-76.

Moraes MEB et al. 2012. Análise da paisagem da bacia hidrográfica do rio Almada (BA) com base na fragmentação da vegetação. Caminhos de Geografia, Uberlândia (MG), 13(41): 159-169.

Moraes MEB et al. 2015. Análise métrica da paisagem na microbacia do rio Água Preta do Mocambo, Uruçuca, 
sul da Bahia. REDE - Revista Eletrônica do Prodema, Fortaleza (CE), 9(1):62-72.

Pires JSR et al. 2004. Avaliação da integridade ecológica em bacias hidrográficas. In: Santos JE et al. (Eds), Faces da polissemia da paisagem: ecologia, planejamento e percepção.SãoCarlos (SP): Rima,p.123-150.

Primack RB e Rodrigues E. 2006. Biologia da conservação. 7 ed. Londrina: Editora Planta, 327p.

Rambaldi DM e Oliveira DAS (Orgs). 2003. Fragmentação de ecossistemas: causas, efeitos sobre a biodiversidade e recomendações de políticas públicas. Brasília: Ministério do Meio Ambiente / Secretaria de Biodiversidade e Florestas, 510 p.

Ranta P et al. 1998. The fragmented Atlantic rainforest of Brazil: size, shape and distribution of forest fragments. Biodiversity and Conservation, 7(3):385-403.

Ribeiro MC et al. 2009. The Brazilian Atlantic Forest: How much is left, and how is the remaining forest distributed? Implications for conservation. Biological Conservation,142(6):1141-1153.

Sambuichi R et al. 2012. Cabruca agroforests in southern Bahia, Brazil: tree component, management practices and tree species conservation. Biodiversity and Conservation, 21(4):1055-1077.

Schroth G et al. 2011. Conservation tropical landscapemosaics: the case ofthecocoaof southern Bahia, Brazil. Biodiversity and Conservation, 2(8):1635-1654.

SEI - Superintendência de Estudos Econômicos e Sociais da Bahia. 2016. Geoinformação: divisão político-administrativa. Disponível em: http://www.sei.ba.gov.br/index.php?option=com content\&view $=$ article\&id $=2396 \&$ Itemid $=728$

Acesso em: 04 nov. 2016.

Silva MSF e Souza RM. 2014. Padrões espaciais de fragmentação florestal na flora do Ibura - Sergipe. Mercator, Fortaleza, 13(3): 121-137.

Viana VM. et al. 1992. Restauração e manejo de fragmentos florestais. Revista do Instituto Florestal, São Paulo, 4:400-407.

Viana WRCC e Moraes MEB. 2016. Bacia do rio Almada (Bahia): criação de cenários ambientais a partir do diagnóstico da fragmentação florestal. In: Moraes MEB e Lorandi R. (Orgs), Métodos e técnicas de pesquisa em bacias hidrográficas. Ilhéus (BA): Editus, p. 35-56.

Tabarelli M e Gascon C. 2005. Lessons from fragmentation research: improving management and policy guidelines for biodiversity conservation. Conservation Biology,19(3):734-739.

Thomas WW et al. 2008. Diversity of woody plants in the Atlantic coastal forest of southern Bahia, Brazil. In: Thomas WW e Britton EG (Eds), The Atlantic Coastal Forests of Northeastern Brazil. New York: The New York Botanical Garden Press. 100: 21-66. 